\title{
Bose-Einstein condensation of a quantum group boson gas
}

\author{
Marcelo R. Ubriaco* \\ Laboratory of Theoretical Physics \\ Department of Physics \\ University of Puerto Rico \\ P. O. Box 23343, Río Piedras \\ PR 00931-3343, USA
}

\begin{abstract}
We study the Bose-Einstein condensation of a gas with $S U_{q}(2)$ symmetry. We show, in the thermodynamic limit, that the boson interactions introduced by the quantum group symmetries enhance Bose-Einstein condensation giving a discontinuity in the heat capacity $C_{v}$ at the critical temperature $T_{c}$. The critical temperature and the gap in $C_{v}$ increase with the value of the parameter $q$ and become approximately constant for $q>3$.
\end{abstract}

PACS number(s): 05.30.-d

*E-mail:ubriaco@ltp.upr.clu.edu 


\section{Introduction}

The search for new applications of quantum groups [1, 2], other than the theory of integrable models, have diversified to several areas of theoretical

physics. The literature on this subject deals mainly with formulations to build quantum group versions of the Lorentz and Poincaré algebras [3], or the use of a quantum group as an internal symmetry in quantum mechanics and field theory [四. Although many of these approaches address interesting theoretical questions, they in general remain at a formalism level, with the quantum group parameter $q$ playing merely the role of a deformation parameter. As it is well known, in the quantum inverse scattering method and vertex models, the parameter $q$ acquires a physical meaning through its relation with Planck's constant and the anisotropy of the lattice respectively. In order to look for the physical role that $q$ could play in other areas of physics, in previous papers [5, 6] we began a study of the thermodynamic properties of quantum group gases, which are the quantum group fermion (QGF) and quantum group boson (QGB) models. These models can be interpreted as either fermion or boson gases with interactions fixed by the quantum group. For reasons of simplicity, we considered for both cases the simplest $S U_{q}(2)$ invariant Hamiltonian. In particular, the QGB model has the interesting property that in two and three spatial dimensions the parameter $q$ interpolates within a wide range of attractive and repulsive systems including the free boson and fermion cases. Therefore, the departure from the value $q=1$, which at the mathematical level implies the noncommutativity of quantum group matrix elements, gives in a simple thermodynamic model, an alternative approach to fractional statistics.

This work is a study on the implications of introducing quantum group 
symmetries in a thermodynamic system at low temperatures, with particular emphasis in the phenomenon of Bose-Einstein condensation. There is, at present, no direct indication that quantum group symmetries are realized in any particular thermodynamic system. At the moment, a quantum group gas is a mathematical model which may or may not explain the behavior of real gases in a particular situation. Nevertheless, by performing the kind of calculations considered here, questions regarding the possible relevance of quantum groups in a thermodynamic system can be answered.

Our starting point is to consider the simplest quantum group, $S U_{q}(2)$, invariant Hamiltonian, and calculate the thermodynamic properties of the corresponding interacting boson gas. Our main motivation resides in finding out about the role that these interactions, which result from the requirement of quantum group invariance, may play at low temperatures. It would be of much interest, in the author's opinion, if these interactions could fit energy related data obtained from the experiments.

In experimental settings, trapping potentials are well approximated by the potential of a harmonic oscillator. A noninteracting Bose gas in a harmonic potential [7] is known to exhibit a discontinuity in the heat capacity. This discontinuity is, however, considerably larger than the one reported in a recent experiment involving a dilute gas of ${ }^{87} R b[8]$. In addition, introducing two-particle interactions, has the effect [9] , for the case of a harmonic oscillator potential, of making the heat capacity continuous, and therefore less agreeable with Reference [8]. Therefore, it is natural to consider whether the boson interactions, required by the quantum group invariance of the Hamiltonian, give a thermodynamic behavior that could fit this and other experimental data. In addition, we expect these interactions to be weak, and a good agreement should occur for values of $q$ close to the standard value 
$q=1$.

In this paper, as a first step, we study the QGB model with no external potential. An obvious question to address is, under which conditions the QGB model exhibits Bose-Einstein condensation (BEC). Under these conditions, what is the behavior of the internal energy and heat capacity at the critical temperature $T_{c}$, and what is the dependence, if any, of $T_{c}$ on $q$. In this paper we answer those questions by analyzing the low temperature behavior of the QGB model with $S U_{q}(2)$ symmetry.

In Section (2) we discuss the general formalism and redefine our model in terms of boson operators. A more detailed and general discussion is given in Reference [6]. Section 3 contains the main results of the paper. We calculate the dependence of the heat capacity $C_{v}$, internal energy $U$, entropy $S$ and equation of state on the parameter $q$. In particular, we show, that the boson interactions that arise from the $S U_{q}(2)$ symmetry of the Hamiltonian, produce a discontinuity, $\lambda$-transition, in the heat capacity at the condensation temperature.

\section{$2 \quad S U_{q}(2)$ bosons}

The quantum group $S U_{q}(2)$ consists of the set of matrices $T=\left(\begin{array}{ll}a & b \\ c & d\end{array}\right)$ with elements $\{a, b, c, d\}$ generating the algebra

$$
\begin{array}{cl}
a b=q^{-1} b a \quad, \quad a c=q^{-1} c a \\
b c=c b \quad, \quad d c=q c d \\
d b=q b d \quad, \quad d a-a d=\left(q-q^{-1}\right) b c \\
\quad \operatorname{det}_{q} T \equiv a d-q^{-1} b c=1,
\end{array}
$$


with the unitary conditions $[10] \bar{a}=d, \bar{b}=q^{-1} c$ and $q \in \mathbf{R}$. Hereafter, we take $0 \leq q<\infty$. The $S U_{q}(2)$ transformation $T$ matrix and the corresponding $R$ matrix

$$
R=\left(\begin{array}{cccc}
q & 0 & 0 & 0 \\
0 & 1 & 0 & 0 \\
0 & q-q^{-1} & 1 & 0 \\
0 & 0 & 0 & q
\end{array}\right)
$$

satisfy the algebraic relations 11

$$
R T_{1} T_{2}=T_{2} T_{1} R,
$$

and

$$
R_{12} R_{13} R_{23}=R_{23} R_{13} R_{12},
$$

where $T_{1}=T \otimes 1$ and $T_{2}=1 \otimes T \in V \otimes V,\left(R_{23}\right)_{i j k, i^{\prime} j^{\prime} k^{\prime}}=\delta_{i i^{\prime}} R_{j k, j^{\prime} k^{\prime}} \in$ $V \otimes V \otimes V$.

We define as $S U_{q}(2)$-bosons the set of fields $\Phi=\left(\begin{array}{c}\Phi_{1} \\ \Phi_{2}\end{array}\right)$, satisfying the relations

$$
\begin{aligned}
\Phi_{2} \bar{\Phi}_{2}-q^{2} \bar{\Phi}_{2} \Phi_{2} & =1 \\
\Phi_{1} \bar{\Phi}_{1}-q^{2} \bar{\Phi}_{1} \Phi_{1} & =1+\left(q^{2}-1\right) \bar{\Phi}_{2} \Phi_{2} \\
\Phi_{2} \Phi_{1} & =q \Phi_{1} \Phi_{2} \\
\Phi_{2} \bar{\Phi}_{1} & =q \bar{\Phi}_{1} \Phi_{2} .
\end{aligned}
$$

Equations (4)-(7) are covariant under the field redefinitions $\Phi^{\prime}=T \Phi$ and $\bar{\Phi}^{\prime}=\bar{\Phi} \bar{T}$ with $T \in S U_{q}(2)$. It is clear that for $q=1$, the operators $\Phi_{j}$ become ordinary bosons $\phi_{j}$.

The operators $\Phi_{j}$ should not be confused with the so called $q$-boson oscillators. A set $\left(a_{i}, a_{i}^{\dagger}\right)$ of $q$-bosons are defined, by the relations [12, 13]

$$
a_{i} a_{i}^{\dagger}-q^{-1} a_{i}^{\dagger} a_{i}=q^{N}, \quad\left[a_{i}, a_{j}^{\dagger}\right]=0=\left[a_{i}, a_{j}\right]
$$


where $N|n\rangle=n|n\rangle$. Several variations of Equation (8) are common in the literature. By taking two sets of $q$-bosons, as defined in Equation (8), it has been shown [12, 14] that the operators

$$
J_{+}=a_{2}^{\dagger} a_{1}, \quad J_{-}=a_{1}^{\dagger} a_{2}, 2 J_{3}=N_{2}-N_{1},
$$

provide a realization of the quantum Lie algebra $s u_{q}(2)$

$$
\left[J_{3}, J_{ \pm}\right]= \pm J_{ \pm}, \quad\left[J_{-}, J_{-}\right]=\left[2 J_{3}\right]
$$

The main distinction between the $\Phi_{j}$ operators with $q$-bosons is that, in contrast to Equations (四)-(可), the algebraic relations in Equation (8) are not covariant under the action of the $S U_{q}(N)$ quantum group matrices. Several studies on the thermodynamics of $q$-bosons, and similar operators called quons [15, have been published [16, 17]. In particular, a system defined by a 'free' Hamiltonian in terms of $q$-oscillators has been shown to exhibit BEC [18, 19]. Certainly, the work devoted to the thermodynamics of $q$-oscillators represents a test on the consequences of modifying boson commutators, according to Equation (\$) and its different versions. On the other hand, the algebraic relations in Equations (4)-(7), with the model discussed in this paper, address the implications that result of imposing quantum group invariance in a thermodynamic system.

The simplest Hamiltonian written in terms of the operators $\Phi_{j}$ is certainly the one that becomes for $q=1$ the free boson Hamiltonian with two species. It is simply written as

$$
\mathcal{H}_{B}=\sum_{\kappa} \varepsilon_{\kappa}\left(\mathcal{N}_{1, \kappa}+\mathcal{N}_{2, \kappa}\right),
$$

where $\left[\bar{\Phi}_{i, \kappa}, \Phi_{\kappa^{\prime}, j}\right]=0$ for $\kappa \neq \kappa^{\prime}$ and $\mathcal{N}_{i, \kappa}=\bar{\Phi}_{i, \kappa} \Phi_{i, \kappa}$. For a given $\kappa$ the $S U_{q}(2)$ bosons are written in terms of boson operators $\phi_{i}$ and $\phi_{i}^{\dagger}$ with usual 
commutation relations: $\left[\phi_{i}, \phi_{j}^{\dagger}\right]=\delta_{i j}$ as follows

$$
\begin{aligned}
& \Phi_{j}=\left(\phi_{j}^{\dagger}\right)^{-1}\left\{N_{j}\right\} q^{N_{j+1}}, \\
& \bar{\Phi}_{j}=\phi_{j}^{\dagger} q^{N_{j+1}}, \quad j=1,2
\end{aligned}
$$

leading to the interacting boson Hamiltonian

$$
\begin{aligned}
\mathcal{H}_{B} & =\sum_{\kappa} \varepsilon_{\kappa}\left\{N_{1, \kappa}+N_{2, \kappa}\right\} \\
& =\sum_{\kappa} \frac{\varepsilon_{\kappa}}{q^{2}-1} \sum_{m=1}^{\infty} \frac{2^{m} \ln ^{m} q}{m !}\left(N_{1, \kappa}+N_{2, \kappa}\right)^{m}
\end{aligned}
$$

where $N_{i, \kappa}$ is the ordinary boson number operator and the bracket $\{x\}=$ $\left(1-q^{2 x}\right) /\left(1-q^{2}\right)$. The grand partition function $\mathcal{Z}_{B}$ is given by

$$
\mathcal{Z}_{B}=\prod_{\kappa} \sum_{n=0}^{\infty} \sum_{m=0}^{\infty} e^{-\beta \varepsilon_{\kappa}\{n+m\}} e^{\beta \mu(n+m)}
$$

which after rearrangement simplifies to the Equation

$$
\mathcal{Z}_{B}=\prod_{\kappa} \sum_{m=0}^{\infty}(m+1) e^{-\beta \varepsilon_{\kappa}\{m\}} z^{m}
$$

where $z=e^{\beta \mu}$ is the fugacity.

\section{Low temperature behavior}

In this Section we study the low temperature (high density) properties of the QGB gas, which is represented by the partition function in Equation (16). In particular, we will study the conditions under which BEC will occur. In the thermodynamic limit we write

$$
\ln \mathcal{Z}_{B}=\ln \left(1+\sum_{n=1}^{\infty}(n+1) z^{n}\right)+\frac{4 \pi V}{h^{3}} \int_{0}^{\infty} p^{2} \ln \left(1+\sum_{n=1}^{\infty}(n+1) e^{-\beta\{n\} \varepsilon} z^{n}\right) d p
$$


where, as in the ideal Bose case, the divergence of the $\mathbf{p}=\mathbf{0}$ term as $z \rightarrow 1$ has been taken into account by splitting $\ln \mathcal{Z}_{B}$ in a single term plus an integration. The series in the integrand of Equation (17) diverges for $z=1$ and $q<1$, giving in this case no transition point. For $q>1$, the series is more convergent than the ideal Bose case, and BEC is expected to occur. The average total number of particles is given by

$$
\begin{aligned}
\langle N\rangle & =\frac{1}{\beta}\left(\frac{\partial \ln \mathcal{Z}_{B}}{\partial \mu}\right)_{T, V} \\
& =\left\langle N_{0}\right\rangle+\frac{4 \pi V}{h^{3}}\left(\frac{2 m}{\beta}\right)^{3 / 2} \int_{0}^{\infty} x^{2} \frac{\sum_{1}^{\infty}(n+1) n e^{-\{n\} x^{2}} z^{n}}{f(z, q)} d x
\end{aligned}
$$

where the function

$$
f(z, q)=1+\sum_{1}^{\infty}(m+1) e^{-\{m\} x^{2}} z^{m} .
$$

Expanding the integrand in powers of $z$ and integrating we obtain

$$
\langle N\rangle=\left\langle N_{0}\right\rangle+\frac{V}{\lambda_{T}^{3}} \sum_{n=1}^{\infty} z^{n} S(n, q),
$$

where $\lambda_{T}=\sqrt{h^{2} / 2 \pi m k T}$ and the coefficient $S(n, q)$ becomes, for $q=1$, $S(n, 1)=2 / n^{3 / 2}$. Therefore, based on standard notation we rewrite Equation (19) as

$$
\langle N\rangle=\left\langle N_{0}\right\rangle+\frac{2 V}{\lambda_{T}^{3}} g_{3 / 2}(z, q),
$$

such that $g_{3 / 2}(1,1)=\zeta(3 / 2)$. In the $q \rightarrow 1$ limit, Equation (20) corresponds to the case of an ideal Bose gas with two species. This model will exhibit Bose-Einstein condensation for those values of the temperature or density such that

$$
\lambda_{T}^{3} \geq 2 \frac{V}{\langle N\rangle} g_{3 / 2}(1, q)
$$


where the critical temperature $T_{c}$ and critical density $\rho_{c}$ are given by the following equations

$$
\begin{gathered}
T_{c}=\frac{h^{2}}{2 \pi m k}\left(\frac{\langle N\rangle}{2 V g_{3 / 2}(1, q)}\right)^{2 / 3}, \\
\rho_{c}=\frac{2 g_{3 / 2}(1, q)}{\lambda_{T}^{3}} .
\end{gathered}
$$

Since for $q>1$ the function $g_{3 / 2}(1, q)<g_{3 / 2}(1,1)$, then the critical temperature $T_{c}$ for this model is larger than the critical temperature $T_{c}^{B E}$ for the ideal Bose gas. For a given density the two temperatures are related by

$$
\frac{T_{c}}{T_{c}^{B E}}=\left(\frac{2.612}{g_{3 / 2}(1, q)}\right)^{2 / 3}
$$

giving then $1 \leq T_{c} / T_{c}^{B E} \leq 2.55$. For the internal energy $U$ we have

$$
\begin{aligned}
U & =\frac{-\partial \ln \mathcal{Z}_{B}}{\partial \beta}+\mu\langle N\rangle \\
& =\frac{4 V}{\sqrt{\pi} \lambda_{T}^{3} \beta} \int_{0}^{\infty} x^{4} \frac{\sum_{1}^{\infty}(n+1)\{n\} e^{-\{n\} x^{2}} z^{n}}{f(z, q)} d x .
\end{aligned}
$$

Expanding the integrand in powers of $z$ leads to the equation

$$
U=\frac{3}{2} \frac{2 V}{\beta \lambda_{T}^{3}} g_{5 / 2}(z, q),
$$

with the identification $g_{5 / 2}(1,1)=\zeta(5 / 2)$.

Figure 1 shows a graph obtained from a numerical calculation of the functions $g_{3 / 2}(1, q)$ and $g_{5 / 2}(1, q)$ for $1 \leq q \leq 6$. These functions decrease from its maximum value $g_{3 / 2}(1,1)=2.612$ and $g_{5 / 2}(1,1)=1.341$ and remain practically constant for $q>3$. 


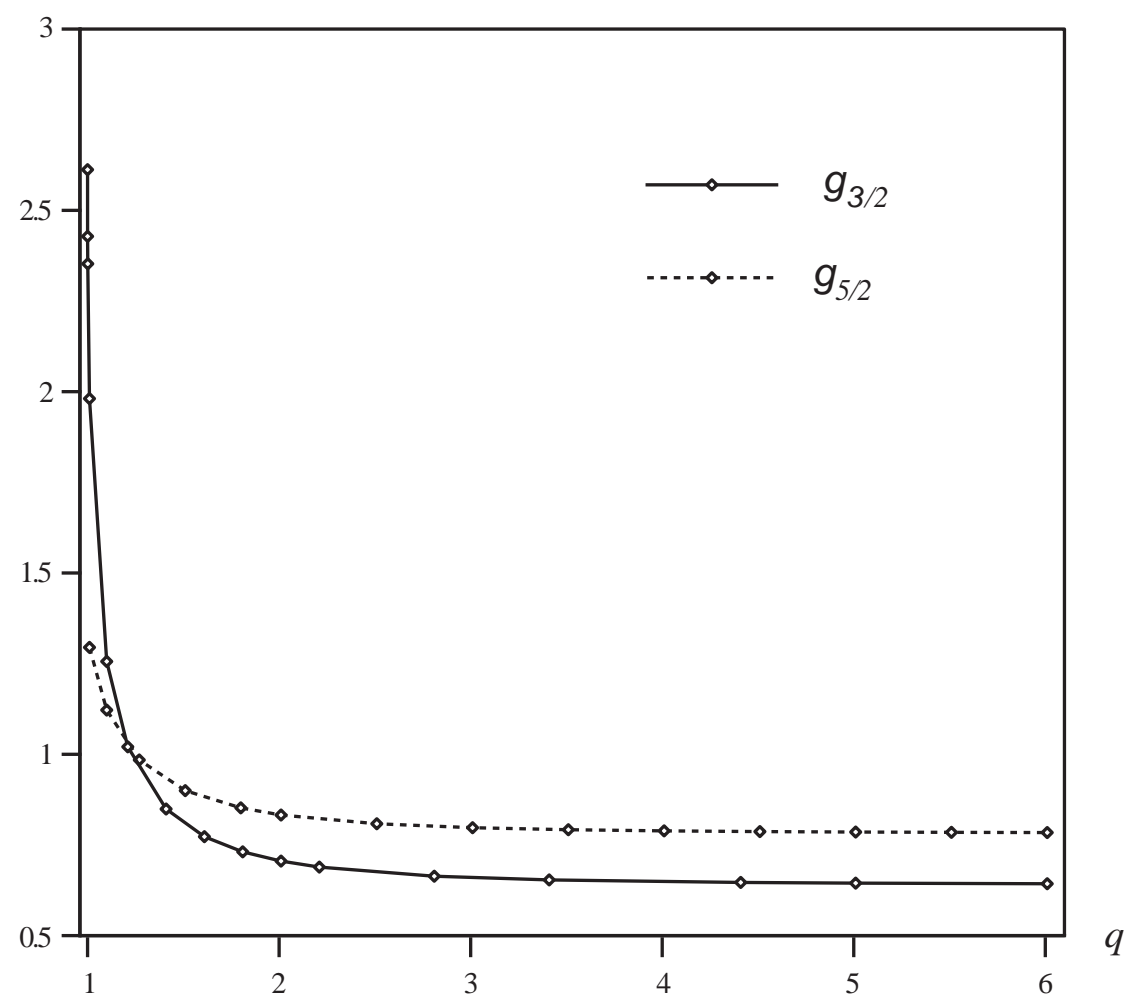

FIG. 1. The functions $g_{3 / 2}(1, q)$ and $g_{5 / 2}(1, q)$, as defined in the text, for $1 \leq q \leq 6$. The value of these functions decrease from its maximum value at $q=1$, and remains approximately constant for $q>3$

The heat capacity is written in terms of the internal energy according to the general expression

$$
\begin{aligned}
C_{v} & =\left(\frac{\partial U}{\partial T}\right)_{V} \\
& =\frac{5}{2} \frac{U}{T}+\left(\beta \mu^{\prime}-\frac{\mu}{k T^{2}}\right) z \frac{\partial}{\partial z} U
\end{aligned}
$$

where $\mu^{\prime}=\partial \mu / \partial T$. For values of the temperature very close to $T_{c}$ the chemical potential $\mu\left(T_{c}^{-}\right)=0$ and its derivative $\mu^{\prime}\left(T_{c}^{-}\right)=0$, and therefore if 
$\mu^{\prime}\left(T_{c}^{+}\right) \neq 0$ there will be a discontinuity in the heat capacity according to

$$
\begin{aligned}
\Delta C_{v} & =C_{v}\left(T_{c}^{-}\right)-C_{v}\left(T_{c}^{+}\right) \\
& =-\beta \mu^{\prime}\left(T_{c}^{+}\right)\left(z \frac{\partial U}{\partial z}\right)_{z=1} .
\end{aligned}
$$

A simple way [9] to find $\mu^{\prime}\left(T_{c}^{+}\right)$is by first considering that for a closed system $\partial\langle N\rangle / \partial T=0$, such that for $T>T_{c},\left\langle N_{0}\right\rangle=0$ so from Equation (20), we obtain

$$
\mu^{\prime}\left(T_{c}^{+}\right)=-\frac{3}{2} k\left(g_{3 / 2}(z, q) / z \frac{\partial g_{3 / 2}(z, q)}{\partial z}\right)_{z=1} .
$$

At $q=1$ the denominator of Equation (29) becomes the divergent series $\sum_{1}^{\infty} k^{-1 / 2}$, and then $\Delta C_{v}=0$. The denominator converges for all values of $q>1$, showing that the heat capacity exhibits a gap at the critical temperature.

In particular, for low temperatures such that $T<T_{c}$ we have that the chemical potential $\mu\left(T<T_{c}\right)=0$ and its derivative $\frac{\partial \mu}{\partial T}\left(T<T_{c}\right)=0$, giving for the heat capacity

$$
C_{v}=\frac{15}{2} \frac{k V}{\lambda_{T}^{3}} g_{5 / 2}(1, q),
$$

such that after inserting Equation (22) the heat capacity becomes

$$
C_{v}=\frac{15}{4} k\langle N\rangle \frac{g_{5 / 2}(1, q)}{g_{3 / 2}(1, q)}\left(\frac{T}{T_{c}}\right)^{3 / 2} .
$$

The dependence of the function $g_{5 / 2} / g_{3 / 2}$ on $q$ will indicate how much the behavior of the heat capacity for this model departs from the ideal Bose case.

In order to find the heat capacity for temperatures $T>T_{c}$, we expand the $\ln \mathcal{Z}_{B}$ in powers of the fugacity $z$. After performing the elementary integrations, the first few terms read

$$
\ln \mathcal{Z}_{B}=\frac{V}{\lambda_{T}^{3}}\left(2 z+4 \delta(q) z^{2}+\ldots\right)
$$


where $\delta(q)=\frac{1}{4}\left(\left[3 /\left(1+q^{2}\right)^{3 / 2}\right]-(1 / \sqrt{2})\right)$. Calculating the average number of particles and reverting the equation, we find, the fugacity and the internal energy as functions of $\langle N\rangle$

$$
\begin{gathered}
z=\frac{\langle N\rangle \lambda_{T}^{3}}{2 V}\left(1-\frac{2 \lambda_{T}^{3} \delta(q)\langle N\rangle}{V}+\ldots\right), \\
U=\frac{3\langle N\rangle}{2 \beta}\left(1-\frac{\lambda_{T}^{3} \delta(q)\langle N\rangle}{V}+\ldots\right) .
\end{gathered}
$$

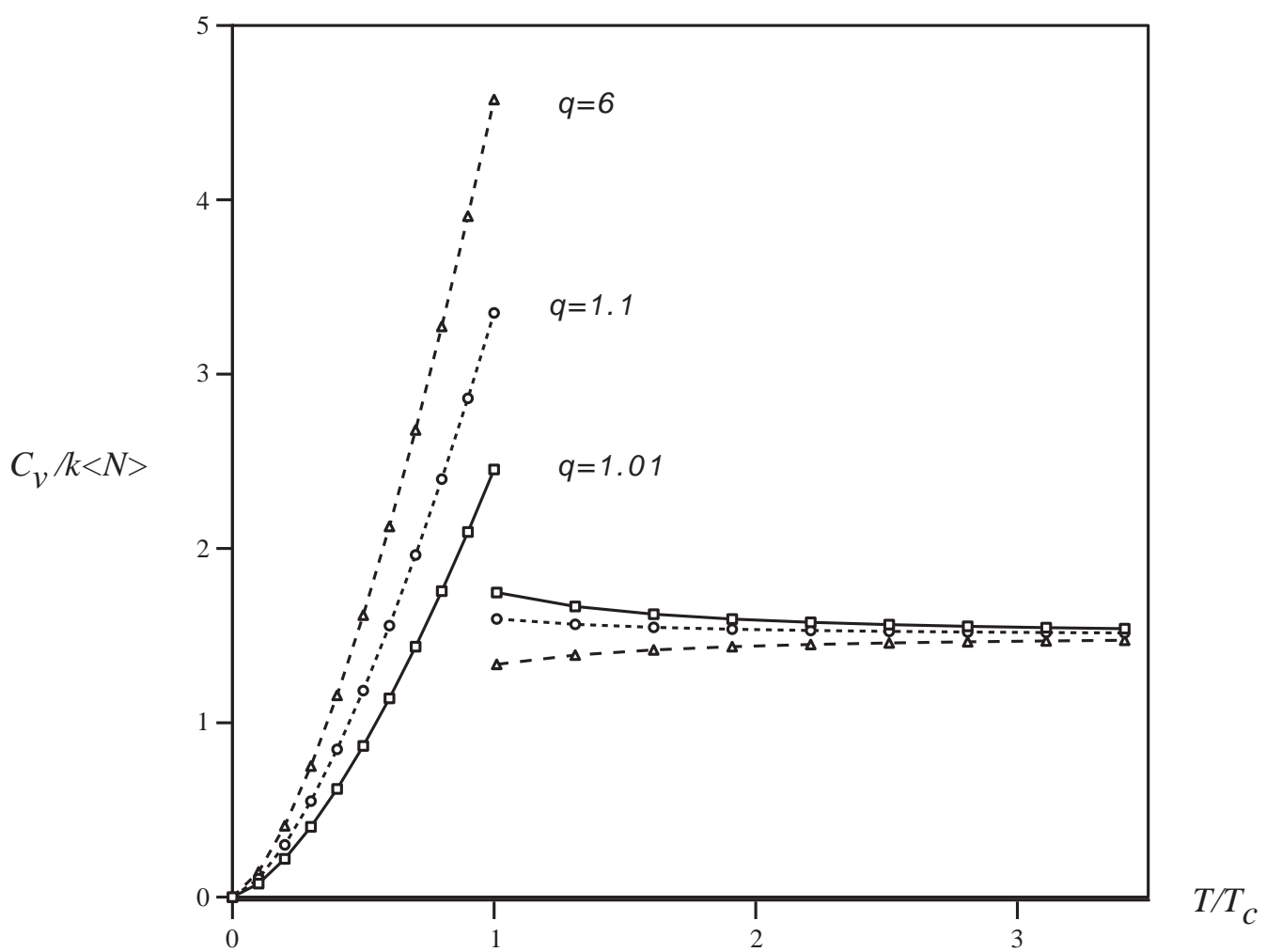

FIG. 2. The heat capacity $C_{v}$ as a function of the temperature for several values of the parameter $q$. The gap at the condensation temperature increases with $q$ up to a value $\Delta C_{v}=3.24 k\langle N\rangle$ at $q=6$ and remains constant for $q>6$. 
The heat capacity is then given by

$$
C_{v}=\frac{3 k\langle N\rangle}{2}\left(1+\frac{\lambda_{T}^{3} \delta(q)\langle N\rangle}{2 V}+\ldots\right),
$$

such that with the use of Equation (22) it becomes

$$
C_{v}=\frac{3 k\langle N\rangle}{2}\left(1+\delta(q) g_{3 / 2}(1, q)\left(\frac{T_{c}}{T}\right)^{3 / 2}+\ldots\right) .
$$

According to Equations (31) and (36) the gap in the heat capacity is then given by the Equation

$$
\left.\Delta C_{v}\right|_{T=T_{c}} \approx k\langle N\rangle\left(\frac{15 g_{5 / 2}(1, q)}{4 g_{3 / 2}(1, q)}-\frac{3}{2}\left(1+\delta(q) g_{3 / 2}(1, q)\right)\right) .
$$

Figure 2 shows the discontinuity of the heat capacity $C_{v}$ for different values of $q$, and shows that the gap is more sensitive to small deviations of $q$ from the ideal Bose case $q=1$. The gap increases with the value of the parameter $q$ and remains approximately constant for $q>3$. Thus, in this model, the onset of BEC becomes a second order phase transition.

At low temperatures $T<T_{c}$, the chemical potential $\mu=0$, giving for the entropy and the equation of state

$$
\begin{aligned}
S & =\frac{U}{T}+k \ln \mathcal{Z}_{B} \\
& =\frac{5 k V}{\lambda_{T}^{3}} g_{5 / 2}(1, q), \\
\frac{p}{k T} & =\frac{2}{\lambda_{T}^{3}} g_{5 / 2}(1, q) .
\end{aligned}
$$

According to Figure 1, the entropy has the interesting property that for a given temperature $T<T_{c}$ it acquires its maximum value at $q=1$. This same feature was also found at low temperatures for the case of a quantum group fermion gas [5]. Then, the interactions introduced by the $S U_{q}(2)$ symmetry are such that they decrease the entropy below the one corresponding to the standard case $q=1$. 


\section{Conclusions}

In this paper we studied the low temperature behavior of a quantum group gas with $S U_{q}(2)$ symmetry in the thermodynamic limit. Our results indicate, that the boson interactions introduced by the quantum symmetry are such that this system exhibits Bose-Einstein condensation for $q>1$.

The interaction terms are fixed by the quantum symmetry of the Hamiltonian, which are the values $N$ and $q$ of $S U_{q}(N)$. For purposes of simplicity, we set $N=2$ and analyzed the effect of varying $q$. The parameter $q$, which in the boson representation controls the strength of the interactions, plays the role of increasing the condensation temperature, reducing the entropy, and producing a gap in the heat capacity at the onset of the BEC. Our results are written in terms of the functions $g_{3 / 2}(1, q)$ and $g_{5 / 2}(1, q)$ introduced in the text. These functions decrease rapidly from their respective values $\zeta(3 / 2)$ and $\zeta(5 / 2)$ at $q=1$, and become approximately constant for $q>3$. Therefore, the properties of this model will be more sensitive to those $q$ values which are small deviations from the ideal Bose case $q=1$. A simple numerical check shows that the critical temperature for $H e^{4}, T_{c} \approx 2.20 \mathrm{~K}$, corresponds to $q=1.02$. Even more interesting is the fact that the same value $q=1.02$ fits very well the gap in the heat capacity of a dilute gas of ${ }^{87} R b$ atoms, as reported in Reference [8], although a realistic comparison with experimental results requires to consider, in addition, an external harmonic potential and the corrections due to a small number $(\approx 20,000)$ of particles. Another interesting feature of this model is that at high temperatures and $q>1.78$ it behaves as an interacting fermion gas [6] and, on the other hand, at low temperatures and $q>1$ it exhibits BEC.

The system studied in this paper is described by the simplest quantum 
group invariant Hamiltonian, and the calculations were made in the thermodynamic limit and with no external potential. Some recent studies for the case of an ideal Bose gas inside a trapping potential, in the thermodynamic limit [9, 20, 21]; and with a finite number of particles [22, 23, 24] show the effect of these corrections on the condensation temperature and heat capacity. Therefore, since in our case, the interactions are fixed by the $S U_{q}(2)$ symmetry of the Hamiltonian, a natural continuation of our work will be to include those corrections such that a more direct comparison to experimental results can be made.

\section{References}

[1] See, for example, M.Jimbo ed., Yang-Baxter equation in integrable systems, Advanced series in Mathematical Physics V.10 (World Scientific,1990).

[2] V. Chari and A. Pressley, A Guide to Quantum Groups, (Cambridge Univ. Press, 1994).

[3] See, for example, U. Carow-Watamura, M. Schlieker, M. Scholl and S. Watamura, Z. Phys. C 48, 150 (1990); O. Ogievetsky, W. Schmidke, J. Wess and B. Zumino, Int. J. Mod. Phys. A 6, 3081 (1990).

[4] I. Aref'eva and I. Volovich, Phys. Lett B 264, 62 (1991); A. Kempf, J. Math. Phys. 35, 4483 (1994); T. Brzezinski and S. Majid, Phys. Lett. B 298, 339 (1993); L. Castellani, Mod. Phys. Lett. A 9, 2835 (1994); M. R. Ubriaco, Mod. Phys. Lett. A 8, 2213 (1993); 10 2223(E) (1995); M. R. Ubriaco, Mod. Phys. Lett. 9, 1121 (1994); A. Sudbery, Phys. Lett. B 375, 75 (1996). 
[5] M. R. Ubriaco, Phys. Lett. A 219, 205 (1996); Mod. Phys. Lett. A 11, 2325 (1996).

[6] M. R. Ubriaco, Phys. Rev. E 55, 291 (1997).

[7] S. R. de Groot, G. Hooyman and C. ten Seldam, Proc. R. Soc. London, Ser. A 203, 266 (1950).

[8] J. Ensher, D. Jin, M. Matthews, C. Wieman and E. Cornell, Phys. Rev. Lett. 77, 4984 (1996).

[9] V. Bagnato, D. Pritchard and D. Kleppner, Phys. Rev. A 35, 4354 (1987).

[10] S. Vokos, B. Zumino and J. Wess, Symmetry in Nature (Scuola Normale Superiore Publ., Pisa, Italy, 1989).

[11] L. A. Takhatajan, Adv. Stud. Pure Math. 19, 1 (1989), and references therein.

[12] A. J. Macfarlane, J. Phys. A 22, 4581 (1989).

[13] L. C. Biedenharn, J. Phys. A 22, L873 (1989).

[14] Y. J. Ng, J. Phys. A 23, 1203 (1990).

[15] O. W. Greenberg, Phys. Rev. Lett. 64, 705 (1990); Phys. Lett. A 209, 137 (1995).

[16] M. Martín-Delgado, J. Phys. A 24,L1285 (1991); I. Lutzenko and A Zhedanov, Phys. Rev. E 50, 97 (1994);P. Angelopoulou,S Baskoutas,L. de Falco,A. Jannussis, R. Mignani and A. Sotiropoulou, J. Phys. A 27, 
L605 (1994); S. Vokos and C. Zachos, Mod. Phys. Lett. A 9, 1 (1994); J. Goodison and D. Toms, Phys. Lett. A 195, 38 (1994); 198, 471 (1995). M. R-Monteiro, I. Roditi and L. Rodrigues, Mod. Phys. Lett. B 9, 607 (1995).

[17] D. Fivel. Phys. Rev. Lett. 65, 3361 (1990). R. Campos, Phys. Lett. A 184, 173 (1994). S. Dalton and A. Inomata, Phys. Lett. A 199, 315 (1995). A. Inomata, Phys. Rev. A 52, 932 (1995).

[18] Gang Su and Mo-lin Ge, Phys. Lett. A 173, 17 (1993); M. R-Monteiro, I. Roditi and L. Rodrigues, Phys. Lett. A 188, 11 (1994.

[19] J.Tuszyński, J. Rubin, J. Meyer and M. Kibler, Phys. Lett. A 175, 173 (1993); M. Salerno, Phys. Rev. E 50, 4528 (1994).

[20] V. Bagnato and D. Kleppner, Phys. Rev. A 44, 7439 (1991).

[21] H. Haugerud and F. Ravndal, cond-mat/9509041.

[22] S. Grossmann and M. Holthaus, Z. Naturforsch 50a, 323 (1995); ibid 50a, 921 (1995); Phys. Lett. A 208, 188 (1995); W. Ketterle and N. J. van Druten, Phys. Rev. A 54, 656 (1996).

[23] W. Mullin, cond-mat/9610005.

[24] H. Haugerud, T. Haugset and F. Ravndal, cond-mat/9605100. 